\title{
Appraisal of methods for predicting the cross-wind response of chimneys
}

\author{
R. V. MILFORD
}

\section{Mr G. M. Pinfold, NCL Consulting Engineers}

Mr Milford has undertaken a formidable and valuable task in comparing the various parameters in some of the more frequently used methods of calculating cross-wind response of chimneys. I endorse Mr Milford's contention that predictions of response are subject to considerable uncertainty and large differences can be obtained from different methods (also by varying input parameters within accepted ranges with any one method). This is not helped by different methods recommending different values for damping, Strouhal number and the like.

50. One method not described by Mr Milford, which is useful insofar as it allows for many of the parameters omitted by other methods, is that published in the Engineering Sciences Data Unit (ESDU) Report 78006. ${ }^{29}$ At NCL, we have recently analysed a number of different chimneys by various methods and have found that the CICIND, ${ }^{30} \mathrm{NBC}^{31}$ and $\mathrm{ESDU}^{29}$ methods all appear to give comparable relationships between response and damping. These methods appear to produce reasonable solutions for broad-band response in turbulent air-stream conditions at critical wind speeds above $12-15 \mathrm{~m} / \mathrm{s}$. Some authorities (such as $\mathrm{NBC}^{31}$ ), however, are recognizing the higher response levels which can pertain to conditions of low turbulence (symptomized by low-level temperature inversions) at low critical wind speeds. Critical wind speeds become lower with taller chimneys and these are the structures becoming most liable to this narrow-band type of response. An added factor is that the upper part of a tall chimney is further away from ground-generated turbulence making for greater correlation lengths of vortex shedding and thence a tendency towards a narrow-band response regime.

51. Until comparatively recently, vortex excitation considerations in reinforced concrete chimneys have been largely ignored on the basis that the damping is comparatively high and there has been, to my knowledge, no published specific evidence of damage or excessive cross-wind oscillation in existing structures.

52. NCL has recently been carrying out studies in connection with assignments on chimneys in Australia and southern Africa and some interesting points have arisen. A single flue concrete chimney some $140 \mathrm{~m}$ high in Australia had been known for some time to oscillate. We were able to make a fairly close estimate of the peak amplitude and of the natural frequency of oscillation, the latter by observations over ten cycles on separate occasions. The calculated critical wind speed 
for the structure was $11 \mathrm{~m} / \mathrm{s}$ and the $x / D$ estimated from observations was $5-6 \%$. This is a significant deflexion for a reinforced concrete chimney but is still small in aerodynamic terms. Even allowing for proximity effects of an upwind stack at a distance of 6.5 chimney diameters, this amplitude is significantly greater than that predicted by the usual turbulent wind-model methods but is close to that obtained using the low wind speed, narrow-band prediction methods in reference 31 (NBC) and of a standard deterministic method ${ }^{32}$ assuming $C_{\mathrm{J}} / \beta_{\mathrm{s}}$ of 21 and a Strouhal number of 0.2 . When this method is used for turbulent wind conditions typical values of $C_{\mathrm{L}} / \beta_{\mathrm{s}}$ used lie in the 13-16 range. We believe that with the current state of knowledge the broad-band stochastic prediction methods (e.g., CICIND) ${ }^{30}$ may, in some wind climates, seriously underestimate the response of tall chimneys having low critical wind speeds.

53. Most large reinforced concrete chimneys in the English-speaking world have been traditionally designed to the American ACl 307-79 specification. ${ }^{33}$ Currently, however, designers are moving towards more sophisticated down-wind design methods and higher allowable stresses. The following example illustrates the need to consider cross-wind effects in some detail. The chimney is $275 \mathrm{~m}$ high by $20.5 \mathrm{~m}$ in diameter and is situated in an area subject to strong low-level temperature inversions. It has a natural frequency of $0.16 \mathrm{~Hz}$, a critical wind speed of $16 \mathrm{~m} / \mathrm{s}$ and a basic wind speed of $41 \mathrm{~m} / \mathrm{s}$ ( $3 \mathrm{~s}$ gust, $10 \mathrm{~m}$ above ground). Table 2 gives the range of base bending moments calculated by current methods. Omitting temperature effects, ACI $307-79^{33}$ allows a working stress in the concrete of approximately 0.2 times the 28-day cube strength; currently designers are using allowable stresses up to 0.3 to 0.4 times the 28 -day cube strength. This example shows that when chimneys are designed for down-wind effects to recently developed methods, a proper assessment of cross-wind effects is important, including fatigue effects, ${ }^{35}$ whereas a design to ACI $307-79^{33}$ might reasonably be expected to cover all eventualities.

54. Paragraph 38 states that the NBC of Canada defines the Strouhal number in terms of the mean hourly wind speed, $\bar{V}$. This is presumably an interpretation of equation (9) of the 1970 edition, ${ }^{36}$ relating frequency of vortex shedding with wind speed. The NBC use of the symbol $\bar{V}$ for wind speed, being the same as that also used in the Code for the mean hourly reference wind speed is, I believe, just an unfortunate coincidence. The 1980 edition $^{31}$ is clearer but the symbol for wind speed $V_{\mathrm{H}}$ is also one used earlier in the document, this time for mean hourly wind speed at the top of the structure. I believe that in both cases the wind speed is

Table 2. Calculated base bending moments

Down-wind

\begin{tabular}{|c|c|}
\hline $\begin{array}{l}\text { Wind loads. (CP3: ch V, Part 2) } \\
\text { Gust energy method (NBC of Canada) } \\
\text { Gust energy method (CICIND) } \\
\text { ACI } 307-79 \text { (lowest allowable wind area) } \\
\text { A3 }\end{array}$ & $\begin{array}{r}820 \mathrm{MNm} \\
830 \mathrm{MNm} \\
680 \mathrm{MNm} \\
1230 \mathrm{MNm}\end{array}$ \\
\hline \multicolumn{2}{|l|}{ Crosswind } \\
\hline $\begin{array}{l}\text { NBC CICIND/ESDU } \\
\text { Deterministic (narrow band) }\end{array}$ & $\begin{array}{c}460 \mathrm{MNm}(x / D=1 \cdot 3 \%) \\
850-1000 \mathrm{MNm} \\
(x / D=2 \cdot 4 \% \text { to } 2 \cdot 8 \%)\end{array}$ \\
\hline
\end{tabular}


intended to be taken as the critical wind speed for resonance.

55. Regarding paragraph 34 , a distinction needs to be made between the distribution along the structure of the forcing function on the one hand and that of the forces which cause the internal actions and stresses on the other. In a lightly damped structure at resonance the former is not really relevant except insofar as it affects the magnitude of the response. The stresses in the structure result principally from the inertial forces resulting from oscillation, the distribution of which is proportional to the product of the mode shape and the mass per unit length. Thus for a chimney having a relatively uniform mass distribution the best estimated equivalent static force for design purposes would be distributed in proportion to the mode shape.

\section{Mr E. Hjorth-Hansen, Norwegian Institute of Technology}

I would like to comment on a side issue of the Paper, and do that with a wider scope than loading models for chimneys in mind. Paragraphs 8 and 34 give the impression that loading models for constant-amplitude, resonant response are arbitrary and dubious-perhaps even in smooth flows where this type of response is encountered. It should be emphasized that complete freedom of choice does not exist for the load distribution function, $\psi$, which is seen by the Author as an arbitrary measure for obtaining a size reduction. The lack of freedom is easily seen when the futile attempt is made to use orthogonal functions for $\psi$ and the mode shapes $\phi$ in equation (9).

57. My preference for a reference load shape $\psi$ is: a function

(a) of the same polarity as the specific mode studied (with the sign function of $\phi$ as one of the simplest choices) in order to make the response unimodal through each of the structural resonances and to avoid widely scattering values of $C_{\mathbf{L}}$ for the successive modes stimulated

(b) equal to zero on parts not exposed to the air flow.

If the first requirement is met, the experimentalist's interpretation of measured response into estimated values for $C_{\mathrm{L}}$ will be of roughly the same size for different modes.

58. The simplest form, $\psi=\operatorname{sign}(\phi)$ (or $\phi$ itself) leads correctly to excitation in all modes, whereas $\psi=1$ (or $\phi$ ) does not necessarily do so. The useful forms mentioned for $\psi$ are capable of taking the place of the popular concept of negative aerodynamic damping, provided of course that the motion-dependency is put into $C_{\mathbf{L}}$.

\section{Mr Milford}

The down-wind and cross-wind comparison drawn by Mr Pinfold is noted with interest, and in particular the results obtained from ACI $307-79^{33}$ which were not considered in the Paper.

60. Referring to paragraph 54 of the discussion, I am not sure if Mr Pinfold's discussion clarifies the issue. At the time of preparing this reply I do not have access to the 1980 edition of the NBC of Canada. ${ }^{31}$ However, referring to the 1977 edition (reference 13 of the Paper), equations (5) and (9) clearly imply that an averaging period of one hour is associated with the critical wind speed. It should be noted that it is not intended to imply in the Paper that the response is dependent on the interpretation of the averaging period associated with the critical wind 
speed, nor should the critical wind speed be converted from one averaging period to another. The discussion on the averaging period refers to the assessment of the return period of the critical wind speed.

61. Paragraphs 8 and 34 of the Paper refer to estimating the cross-wind displacement of the chimney and not stress-resultants. Mr Pinfold notes that the internal actions and stresses should strictly be obtained from the distribution of the inertia forces, and not on the distribution of the aerodynamic forcing function. (For chimneys vibrating in the fundamental mode the latter approach is generally conservative.) However, the distribution of the equivalent aerodynamic force used in an equivalent static load design approach is independent of the mode shape, and should be chosen in conjunction with the aerodynamic parameters used and experimental results. As shown in the Paper, while keeping the other parameters constant, the magnitude of the response is dependent on the distribution function used.

62. Finally, to my knowledge, the ESDU Report No $78006^{29}$ is based on the earlier work of Vickery which has since been updated and which has been discussed in detail in the Paper.

63. As noted by $\mathrm{Mr}$ Hjorth-Hansen, the load distribution function is not arbitrary - the use of a load distribution function orthogonal to the mode shape would not only be futile but also unrealistic. Rather, the load distribution function should be chosen intuitively, or based on experimental results. $\mathrm{Mr}$ HjorthHansen's choice of the mode shape for the distribution function is the same as that of reference 26 of the Paper and in keeping with paragraph 55 of Mr Pinfold's discussion.

\section{References}

29. EngineERING SCIENCEs Data Unit. Across-flow response due to vortex shedding. ESDU, London, 1978, Report no. 78006.

30. Comite International des Cheminés Industrielles Proposal for a model code for the design of chimneys. CICIND, 1981.

31. National Building Code of Canada. Commentary B, wind loads 1980, National Research Council of Canada, Ottawa, 1980.

32. Pinfold G. M. Reinforced concrete chimneys and towers. Cement and Concrete Association, Wexham Springs, 1975, Viewpoint publication.

33. AMERICAN CONCRETE INSTITUTE. Specification for the design and construction of reinforced concrete chimneys. ACI, Detroit, 1979, ACI 307-79.

34. British Standards Institution: Wind loads. BSI, London, 1972, CP3: Ch. 5, Part 2.

35. Bell M. H. and Shears M. Assessment of wind effects in design practice. Wind engineering in the Eighties, Construction Industry Research and Information Association, London, 1980, Paper No. 14.

36. National Building Code of Canada. Commentary no. 1 of the National Building Code of Canada 1970. National Research Council of Canada, Ottawa, 1970. 